PS04.04.24 CRYSTAL STRUCTURE OF THE B-DNA DECAMER D(CGCAATTGCG) $)_{2}$; SEQUENCE-DEPENDENT CROSSED HELIX PACKING. Alexis A. Wood, Christine M. Nunn, Stephen Neidle, The CRC Biomolecular Structure Unit, The Institute of Cancer Research, Cotswold Road, Sutton, Surrey, SM2 SNG, UK

The B-DNA decamer d(CGCAATTGCG $)_{2}$ has been found to crystallise in the monoclinic space group $\mathrm{C} 2$ and packs with a crossed arrangement of helices and a unique crossing contact distinct from all other decamer structures. This is believed to be a direct result of the sequence-dependent conformational features of the duplex. Two unique sites are observed at the cross-over junction where strand exchange may occur if this structure were to be used as a model for the Holliday juction. It is also a rare example of a B-DNA sequence crystallising in two different space groups by variation of crystallisation conditions alone. The structure has been solved to a resolution of $2.3 \AA$ and an $\mathrm{R}$-factor of $17.7 \%$.

MS04.04.25 BEYOND A, B AND Z-DNA. Olga Kennard, Cambridge Crystallographic Data Centre, 12 Union Rd. Cambridge, CB2 IEZ, UK

During the past decade single crystal $x$-ray analysis has led to the characterisation of a number of DNA motifs in addition to the classical $A$. $B$ ans $Z$ duplexes. These include examples, by several authors, arising from hydrogen bonding between looped out bases of neighbouring duplexes. We have made use of such interactions to engineer fragments of the triple helix in two crystal structures. Other experiments, principally by Rich and co-workers, were based on crystallising sequences with specialised biological function, where duplex formation was excluded. These have confirmed and extended structural information on the I-motif of intercalated C-C pairs and the G-tetrad. Another, unexpected quadruplex motif was found in crystals of a potentially self-complementary sequence and the most recently has reappeared in an unrelated cyclic oligonucleotide. A combination of carefully designed experiments and the occasional unexpected result has taken our knowledge of DNA structure beyond the double helix.

\section{Protein- DNA}

MS04.05.01 DNA STRUCTURE AND PROTEIN-DNA INTERACTIONS. C.R. Calladine, Department of Engineering, University of Cambridge, Cambridge CB2 1PZ, UK

When protein binds to DNA, the DNA is sometimes distorted and sometimes not: evidently the deformability of DNA is a feature of the recognition process. What is the general nature of distortion of and within DNA? And how does the presence of a protein impose such distortion? Now, the various components of DNA are connected together in a wellknown way : the base-pairs stack onto each other, and they are connected to the double-helical backbones. The local and global conformation of the molecule may be described by means of a large number of geometric parameters; and in general the values of these must change when the conformation alters. But what are the physically important features of these geometric changes?

In this talk I shall examine the stacking preferences of the 10 different dinucleotide steps by sequence (mainly by a detailed inspection of the variety of step conformations found in a crystallographic database) and show how these relate to larger-scale geometric features of the DNA such as curvature, twist and the widths of the major and minor grooves. I shall also examine the systems of constraint that are necessary in order to "dock" a protein onto a piece of DNA so that the protein side-chains may "probe" the details of the bases concerned. This will include a discussion of the role of "flexible" protein moieties in the business of recognition. References

1. C.R. Calladine \& H.R. Drew. Understanding DNA. Academic Press 1992. 2. M.A. El Hassan \& C.R. Calladine. J. Mol. Biol. (1995) 251, 648-664; and in press.
MS04.05.02 LAC REPRESSOR: STRUCTURE AND GENETICS. M. Lewist, M. A. Kercher", G. Changt, N. C. Horton $^{*}$, J. H. Miller + , H. C. Pace ${ }^{*}$, and P. Lu* ${ }^{*}$ † Department of Biochemistry and Biophysics and "Department of Chemistry, University of Pennsylvania, Philadelphia, PA 19104, USA and †Department of Microbiology and Molecular Genetics, University of California, Los Angeles, CA 90024, USA

The lac operon of $E$. coli is the paradigm for gene regulation. The key component of the operon is the lac repressor, a product of the lacI gene. The 3-dimensional structures of the intact lac repressor, the lac repressor bound to the gratuitous inducer isopropyl-1- $\beta$-D-thiogalactoside (IPTG) and the lac repressor complexed with a 21 base-pair symmetric operator DNA have been determined. These three structures show the conformation of the molecule in both the induced and repressed states and provide the framework for understanding a wealth of biochemical and genetic information. A comprehensive site specific substitution analysis on the lac repressor protein has resulted in over four thousand single amino acid replacements of known phenotype between codons 2 through 329 of the lacI gene. We have made an initial correlation of the site-specific mutational analysis with the 3dimensional structures. In addition, the DNA sequence of the lac operon has three lac repressor recognition sites in a stretch of 500 base pairs. The crystallographic structure of the complex with DNA suggests that the tetrameric repressor functions synergistically with catabolite gene activator protein (CAP) and is involved in the quaternary formation of repression loops in which one tetrameric repressor interacts simultaneously with two sites on the genomic DNA. (supported by NIH and US ARO)

MS04.05.03 MECHANISM OF PYRIMIDINE DIMER EXCISION REPAIR: STRUCTURAL BASIS OF DAMAGED DNA RECOGNITION. K. Morikawa* D.G. Vassylyev", , T. Kashiwagi", Y. Mikami", M. Ariyoshi", S. Iwai", E. Ohtsukat, "Biomolecular Engineering Research Institute, 6-2-3, Furuedai, Suita, Osaka, 565, Japan; +Faculty of Pharmaceutical Sciences, Hokkaido Univ., Sapporo, Hokkaido, 060, Japan

The crystal structure of $\mathrm{T} 4$ endonuclease $\mathrm{V}$ (inactive mutant) in complex with DNA substrate $(12$ bp) has been solved at $2.75 \AA$ resolution. The atomic model of the complex reveals the unique conformation of the B-DNA duplex with a sharp (60 degrees) kink at the central pyrimidine dimer (PD). The adenine base complementary to $5^{1}$ side of the PD moiety is completely flipped out of the DNA duplex and accomodated into a cavity on the enzyme surface. The kink induces the deformation of the phosphate backbone in the vicinity of $\mathrm{PD}$. This deformation is recognized by the protein, instead of unique architecture of PD base moiety. Such indirect readout of DNA damage may be general for the DNA repair mechanisms with broad specificity. In addition to the contribution to the protein/DNA recognition the flipped out base also generates an empty space inside DNA which allows the access of the catalytically active residues to the target bond of PD. Thus flipped out base is likely to be a common feature of other DNA glycosylases/DNA complexes.

Crystals of the complex belong to the space group $P 65(a=b=118.8 \AA, c=36.3 \AA, Z=6)$. The structure was solved by MR method combined with solvent flattening and refined at $2.75 \AA$ resolution to a final $R$-factor of 0.152 ( $R$-free $=0.248$ ). 